\title{
La médecine, les médecins et les juges...
}

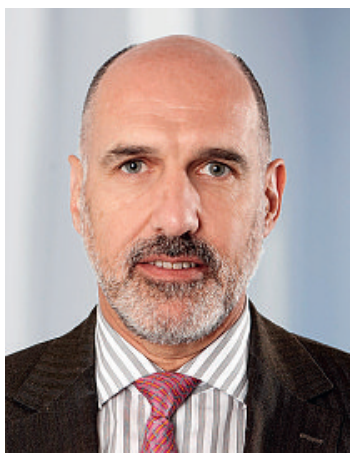

Vous l'avez certainement lu ou entendu: le Tribunal fédéral, selon le communiqué [1] qu'il a publié fin août à ce sujet, estime qu'un prisonnier qui fait une grève de la faim doit être alimenté de force si sa santé est en danger.

Alimenté par qui? Ce n'est pas précisé dans le communiqué, mais il est clair pour tout le monde que les médecins en charge du prisonnier vont se trouver chargés là, par les autorités, d'un mandat qui risque d'être simplement incompatible avec notre déontologie, avec l'éthique médicale.

\section{Les médecins en charge du prisonnier vont se trouver chargés d'un mandat incompatible avec notre déontologie}

En effet, notre code de déontologie est sans équivoque: la décision d'un prisonnier gréviste de la faim qui, lucidement et en connaissance de cause, prend le risque de la mort, doit être respectée par le médecin en charge [2]. Autrement dit, la liberté d'un patient, d'une patiente de refuser un traitement est une liberté qui doit absolument être défendue en toute circonstance, et l'emprisonnement n'y change rien:
Le corps médical ne peut se taire lorsqu'on s'attaque à la relation du médecin avec un patient, à la liberté de ce dernier face à un projet thérapeutique, c'est-à-dire à l'autonomie et à la dignité de la personne (même emprisonnée, bien sûr), et ainsi finalement à la base fondamentale de notre travail.

Une interférence extérieure judiciaire ou administrative (ou d'ailleurs aussi économique) dans la relation thérapeutique peut rendre celle-ci en grande partie inopérante, et nous ne pouvons l'accepter: cela met en cause non seulement la qualité, mais surtout la nature même de notre travail.

Autrement dit, nous ne pouvons accepter que la médecine soit niée dans sa spécificité et qu'une autorité administrative ou judiciaire prétende, dans les faits, exiger une médecine à son service, une médecine sans relation vraie, purement mécanique, purement opérative.

Concrètement, il est clair qu'un médecin de prison chargé d'une tâche de police, qui plus est potentiellement physiquement violente (il faut attacher le prisonnier pour le nourrir de force!), est alors assimilé à l'institution par le prisonnier-patient et ne peut plus avoir avec lui la relation de confiance qui seule, nous le savons tous, permet une médecine authentique.

C'est un fait: l'inclusion du médecin dans des actions de nature sécuritaire n'est pas compatible avec l'exercise de la médecine en milieu pénitentiaire.

On nous a dit: «Mais vous devez nourrir ce prisonnier même de force, il n'est pas sûr qu'il veuille vraiment mourir!» Or justement, comment le savoir si ce n'est au travers d'une relation véridique, dénuée d'interférences et de violence potentielle? En voulant dénaturer la médecine, la justice se

Nous ne pouvons accepter que la médecine soit niée dans sa spécificité et qu'une autorité prétende exiger une médecine à son service

les entraves à la liberté d'un prisonnier ne peuvent d'aucune façon ni interférer avec la relation médicale ou avec la qualité du traitement dont il peut avoir besoin, ni toucher à sa liberté de disposer de sa vie.

Telle qu'elle est soudain exigée par le Tribunal fédéral, l'instrumentalisation des médecins par l'autorité a beaucoup fait parler d'elle dans les médias, et aussi soulevé des vagues importantes dans les milieux médicaux concernés. Nous y consacrons donc une large place dans le présent numéro du Bulletin des médecins suisses.

Pourquoi ces vagues, et pourquoi est-ce important?

Parce que le Tribunal fédéral touche ici de façon inconsidérée à un élément central de la médecine, et aussi à la dignité de la personne dans notre société - et ce n'est donc pas rien! tire une balle dans le pied, si l'on ose dire, et se prive du seul accès authentique à la personne en cause!

Cette décision du Tribunal fédéral prouve une fois encore que le corps médical a la tâche, lorsque c'est nécessaire, de rappeler certaines valeurs fondatrices de notre culture. Nous le faisons avec d'autant plus de vigueur que cela touche à la racine de notre travail, et que le corps médical tient assurément là l'une de ses raisons d'être dans la société.

Dr Jacques de Haller, Président de la FMH

1 www.bger.ch/fr/mm_6b_599_2010_d_internet.pdf

2 Annexe 1 au Code de déontologie, voir www.fmh.ch $\rightarrow$ FMH $\rightarrow$ Bases légales $\rightarrow$ Code de déontologie 